\title{
Editorial
}

\section{High-Entropy Alloys}

\author{
Yong Zhang, ${ }^{1}$ Jien-Wei Yeh, ${ }^{2}$ Jian F. Sun, ${ }^{3}$ Jun P. Lin, ${ }^{1}$ and Ke-Fu Yao ${ }^{4}$ \\ ${ }^{1}$ Beijing University of Science and Technology, Beijing 100083, China \\ ${ }^{2}$ National Tsing Hua University, Hsinchu 30013, Taiwan \\ ${ }^{3}$ Harbin Institute of Technology, Harbin 150001, China \\ ${ }^{4}$ Tsinghua University, Beijing 100084, China \\ Correspondence should be addressed to Yong Zhang; drzhangy@ustb.edu.cn
}

Received 18 February 2015; Accepted 18 February 2015

Copyright (C) 2015 Yong Zhang et al. This is an open access article distributed under the Creative Commons Attribution License, which permits unrestricted use, distribution, and reproduction in any medium, provided the original work is properly cited.

High-entropy alloys are usually defined as metallic materials with high entropy of mixing at their disordered state. They tend to form high-entropy disordered solid solutions and amorphous phases and get simpler microstructure. High-entropy alloys can include disordered solid solution alloys and high-entropy metallic glasses. In addition, highentropy ceramics based on the same "high entropy" concept can include carbides, nitrides, borides, and oxides. Recent advances in high-entropy alloys and ceramics have brought new conceptions to develop advanced materials, which may potentially overcome the challenges in increasing the property limits for the advanced energy and environment friendly materials. Knowledge and understanding of these high-entropy alloys and ceramics have led to the usage of novel tools for more detailed study such as serration and crackling noise detection, high energy X-rays synchrotron, neutron diffraction, focus ion beam (FIB), 3-dimensional atomic probe tomography (3D APT), density functional theory modeling (DFT), CALPHAD, and ab initio molecular dynamics (AIMD) modeling.

In this special issue, investigators contribute original research articles as well as review articles that will stimulate the continuing efforts to understand the structure, phase formation rules, thermodynamics, kinetics, processing, and properties underlying the development strategies and the evaluation of outcomes. We are particularly interested in articles describing the new technologies applied for fabricating the materials and characterizing the structural change in the micro- and nanoscale by in situ observations.
The following areas are covered in this special issue: recent developments in high-entropy alloys, ceramics, and amorphous alloys; advances in mechanical and physical behaviors of high-entropy alloys; latest technologies for fabricating and characterizing high-entropy alloys; and serration behaviors and crackling noise of advanced materials.

Yong Zhang Jien-Wei Yeh Jian F. Sun Jun P. Lin $\mathrm{Ke}-\mathrm{Fu} \mathrm{Yao}$ 

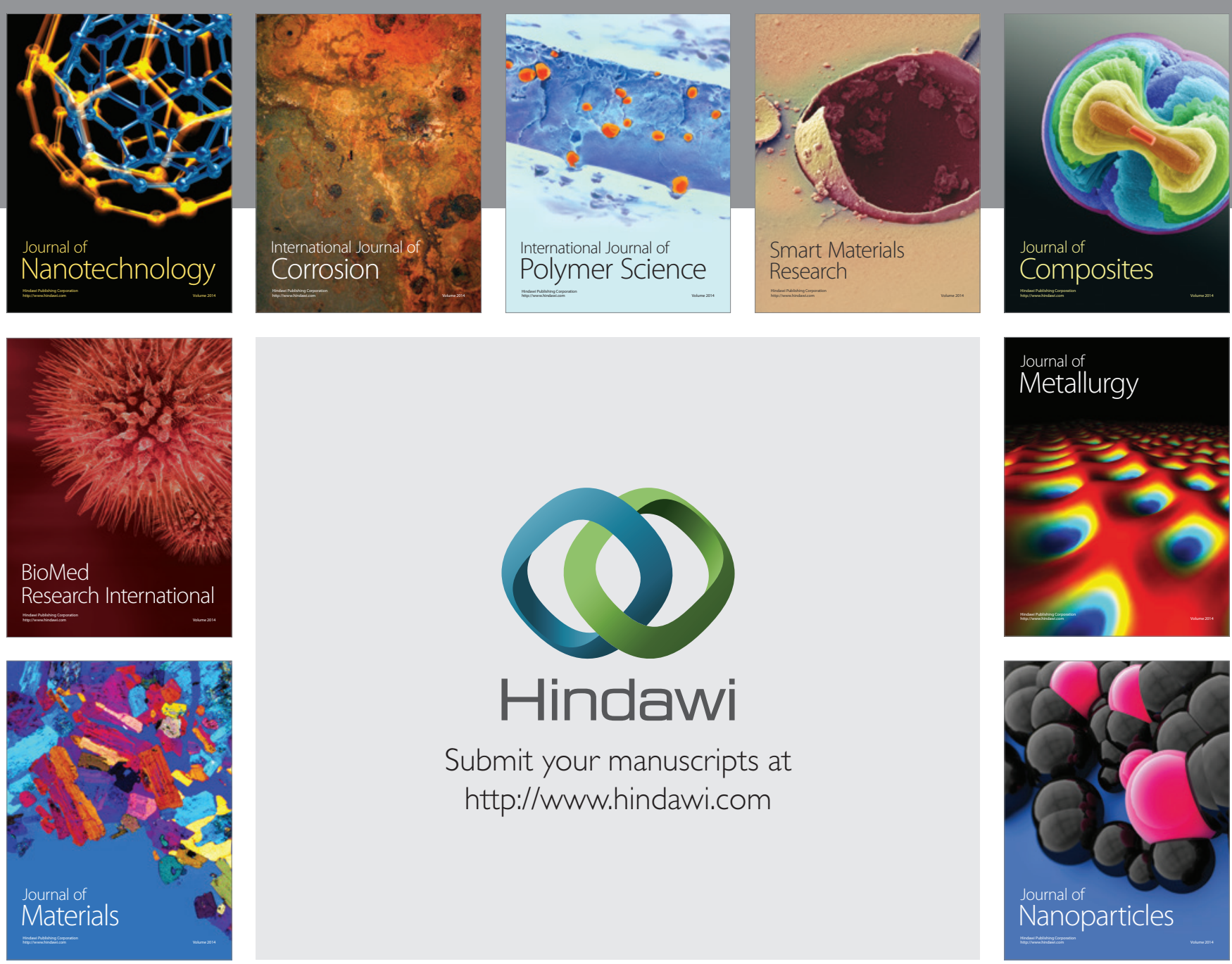

Submit your manuscripts at http://www.hindawi.com
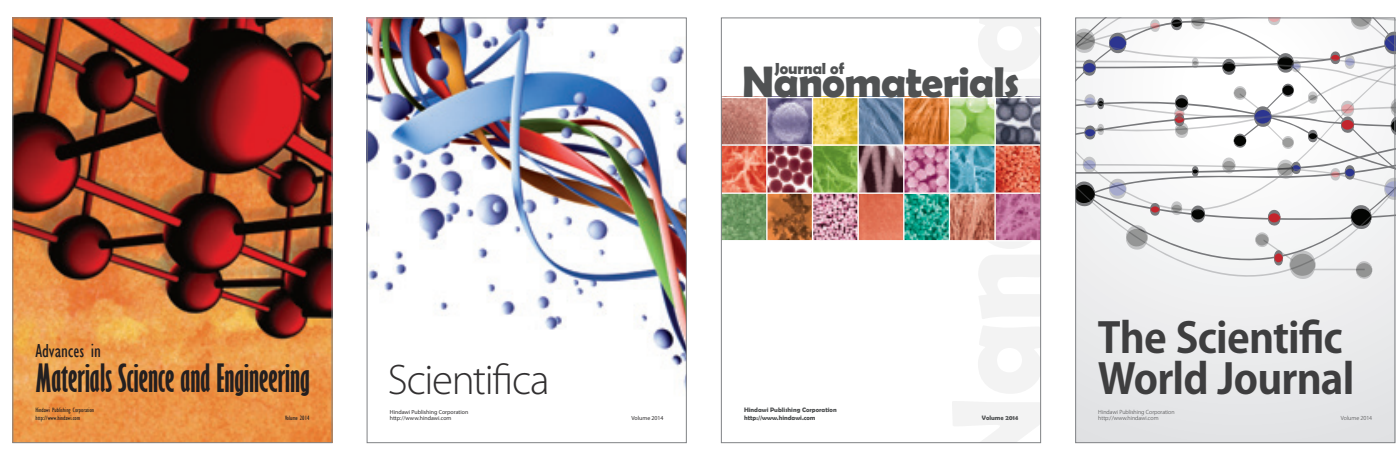

\section{The Scientific World Journal}
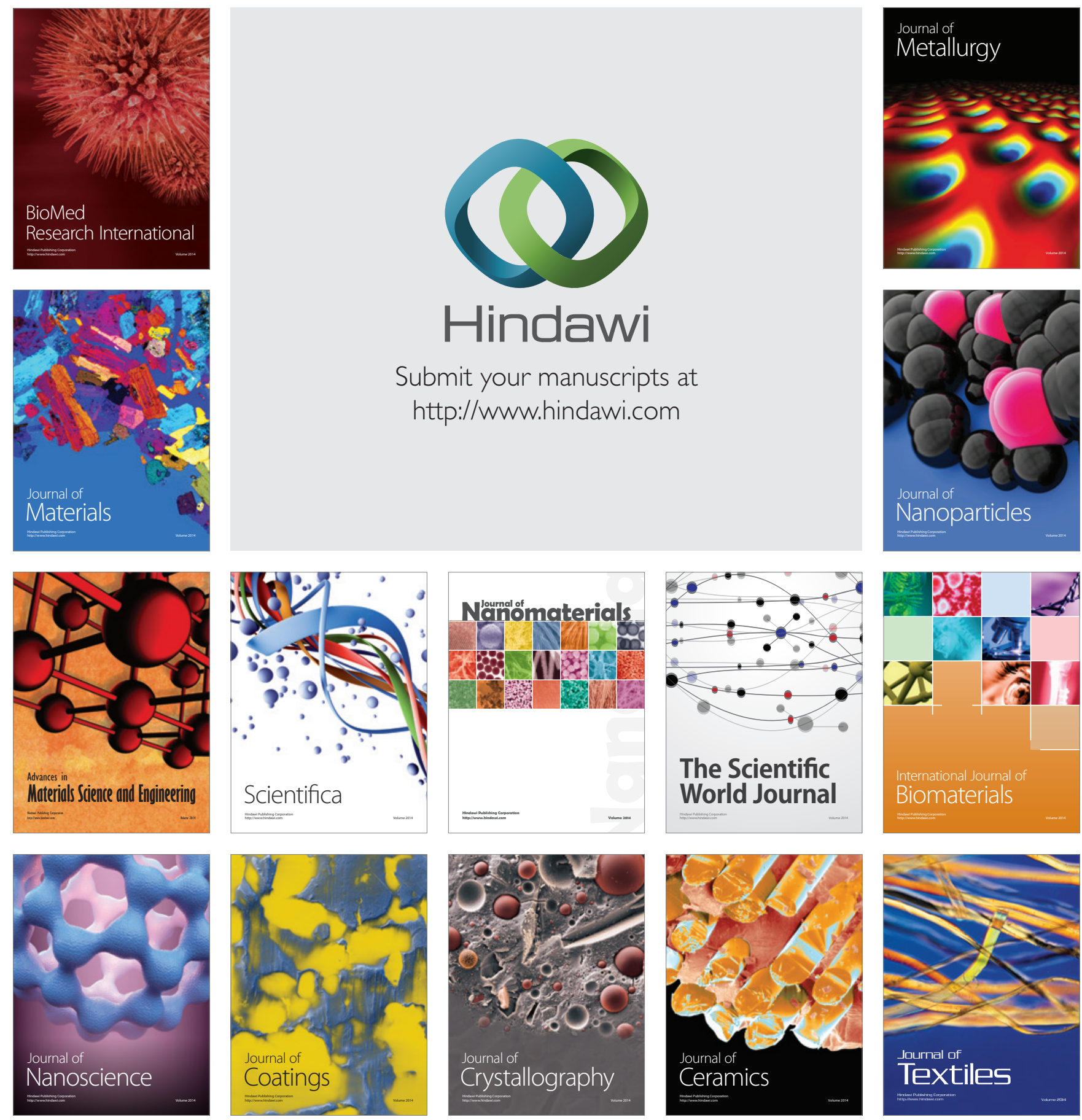